CLINICAL STUDY

\title{
Decrease in circulating fibroblast growth factor 21 after an oral fat load is related to postprandial triglyceride-rich lipoproteins and liver fat
}

\author{
Niina Matikainen ${ }^{1,2}$, Marja-Riitta Taskinen ${ }^{1}$, Sanna Stennabb ${ }^{1}$, Nina Lundbom ${ }^{3}$, Antti Hakkarainen ${ }^{3}$, \\ Kirsi Vaaralahti ${ }^{4}$ and Taneli Raivio ${ }^{4,5}$ \\ ${ }^{1}$ Division of Cardiology, ${ }^{2}$ Division of Endocrinology, Department of Medicine, Helsinki University Central Hospital, University of Helsinki, PO Box 700 , \\ FIN-O0029 Helsinki, Finland, ${ }^{3}$ Department of Radiology, ${ }^{4}$ Children's Hospital, Helsinki University Central Hospital and ${ }^{5}$ Institute of \\ Biomedicine/Physiology, Biomedicum Helsinki, University of Helsinki, FIN-O0029 Helsinki, Finland \\ (Correspondence should be addressed to N Matikainen at Division of Endocrinology, Helsinki University Central Hospital; Email: niina.matikainen@hus.fi)
}

\begin{abstract}
Background: Elevated levels of circulating fibroblast growth factor 21 (FGF21) are commonly encountered in type 2 diabetes, dyslipidemia, and non-alcoholic fatty liver disease, all of which share exaggerated postprandial lipemia as a common proatherogenic feature. How FGF21 responds to an oral fat load in man is unknown.

Methods: We measured liver fat contents and subcutaneous and visceral fat volumes in 47 healthy subjects, who also underwent an oral fat load with measurements of plasma FGF21 and free fatty acid (FFA). Triglyceride (TG), apolipoprotein B-48 (apoB-48), and apoB-100 concentrations were measured in triglyceride-rich lipoprotein (TRL) fractions.

Results: When compared with fasting levels, the concentration of FGF21 decreased significantly at $4 \mathrm{~h}$ $(P<0.05)$ and tended to return to fasting levels at $8 \mathrm{~h}$ after an oral fat load. Fasting and postprandial FGF21 correlated significantly with liver fat as well as with TRLs in the chylomicron and especially in very low-density lipoprotein 1 (VLDL1) and VLDL2 fractions representing remnant particles, but not with FFA. Subjects with increased liver fat $(>5 \%, n=12)$ showed impaired suppression of FGF21 at $4 \mathrm{~h}(P<0.05)$ and at $8 \mathrm{~h}(P=0.01)$ and demonstrated higher postprandial TG area under the curve in plasma and TRL fractions $(P \leq 0.032)$ compared with those with normal liver fat $(\leq 5 \%, n=35)$. Conclusions: We observed a significant decrease of FGF21 concentration after an oral fat load. Fasting and postprandial FGF21 levels were closely related to large VLDL and remnants, but not to plasma FFA. Our pilot findings suggest that the postprandial accumulation of TRL remnants and liver fat may modulate postprandial FGF21 levels.
\end{abstract}

European Journal of Endocrinology 166 487-492

\section{Introduction}

Fibroblast growth factor 21 (FGF21) is considered to act as an endocrine/paracrine feedback signal regulating energy balance. Experimental and animal data demonstrate that during fasting or ketogenic diet, an increase of FGF21 mediates a shift in energy balance in hepatocytes, adipocytes, and islet $\beta$-cells (1). Free fatty acids (FFAs) bind to hepatic peroxisome proliferatoractivated receptor $\alpha(\mathrm{PPAR} \alpha)$, activate FGF21 expression, and thereby increase hepatic fatty acid oxidation (2). In adipocytes, FGF21 enhances mitochondrial oxidative capacity through PPAR $\gamma$ coactivator $1 \alpha$ (3). Because of the observed reductions in weight, glucose, and lipid levels, the FGF21 actions in animals are considered metabolically beneficial (4). However, in man, the physiological function of FGF21 remains under debate.

Fasting FGF21 levels are highly variable among healthy subjects (5). During a 24-h fast, FGF21 levels remain rather stable and do not demonstrate any diurnal variation. Contrary to the findings in rodents, FGF21 is not considered as a vital hormonal switch of nutritional status in humans $(5,6)$. During day-long ingestion of sequential standardized meals, the FGF21 levels peak in the early morning after an overnight fast. Thereafter, FGF21 levels oscillate parallel to the changes in FFA and cortisol concentrations, but in opposite directions to changes in glucose and insulin concentrations (7).

FGF21 levels are increased in dysmetabolic conditions including the metabolic syndrome, obesity (8), insulin resistance (9), type 2 diabetes, and non-alcoholic fatty 
Table 1 Metabolic characteristics and body composition of the study subjects, $n=47$. Data are mean \pm s.D. or interquartile range.

\begin{tabular}{ll}
\hline & Mean \pm s.o. \\
\hline Age (years) & $42.7 \pm 11.7$ \\
Gender (males/females) & $22 / 25$ \\
Body mass index (BMl; kg/m $\left.{ }^{2}\right)$ & $25.9 \pm 4.1$ \\
Liver fat (\%) & $4.2 \pm 5.6$ \\
Subcutaneous fat $\left(\mathrm{cm}^{2}\right)$ & $3939(2409)$ \\
Visceral fat $\left(\mathrm{cm}^{2}\right)$ & $1495 \pm 969$ \\
Glucose $(\mathrm{mmol} / \mathrm{l})$ & $5.23(0.5)$ \\
Glycohemoglobin A1C $(\%)$ & $5.5 \pm 0.4$ \\
LDL cholesterol $(\mathrm{mmol} / \mathrm{l})$ & $3.42 \pm 1.11$ \\
HDL cholesterol $(\mathrm{mmol} / \mathrm{l})$ & $1.43 \pm 0.38$ \\
Triglycerides $(\mathrm{mmol} / \mathrm{l})$ & $1.49(0.98)$ \\
ALT (U/l) & $31(24)$ \\
\hline
\end{tabular}

liver disease (NAFLD) $(8,10)$. Likewise, in subjects with dyslipidemia and coronary heart disease, elevated concentrations of triglycerides (TGs) and apolipoprotein A1 (apoA1) independently predicted elevated FGF21. A common feature for these conditions is prolonged and exaggerated postprandial lipemia (11). So far, the relationship between TG metabolism and FGF21 has been poorly characterized.

Increases in adipose tissue lipolysis and, consequently, in FFA concentration during fasting, induce FGF21 expression (4). During alimentary lipemia, fatty acids from the meal are carried as TGs within triglyceride-rich lipoproteins (TRLs) together with hepatic-derived particles, both contributing to the rise in chylomicron, very low-density lipoprotein 1 (VLDL1), and VLDL2 particles (i.e. TRLs) for hours. We hypothesized that ingestion of fat in a form of mixed meal could be reflected to postprandial FGF21 levels through changes in concentrations of either FFA or TRLs, or both.

\section{Subjects and methods}

We recruited 47 previously healthy family members of our dyslipidemic cohort taking part in our previous studies. Of the 54 volunteers screened for the study, we excluded one because of diabetes and six subjects declined to participate in the study because of personal reasons. The subject characteristics are shown in Table 1. To detect possible changes in postprandial FGF21, we included both normolipidemic and mildly dyslipidemic subjects who were otherwise healthy. Further inclusion criteria included patients aged 18-65 years, non-smokers, and those with no history of cardiovascular or other disease. The subjects were screened to confirm they had no secondary conditions affecting the lipid levels or abnormalities in thyroid and kidney function or in basic blood count. Severe liver diseases including non-alcoholic steatohepatitis were excluded by clinical examination and measurement of liver enzymes. Subjects were asked to discontinue possible lipid-lowering therapy 1 month before the study. We performed an oral fat load and determined the liver fat content and the amount of subcutaneous and visceral fat for each study subject. The study was approved by the Ethical Committee of Helsinki University Central Hospital and the study subjects gave their informed consent.

As the liver fat content is a predictor of both hepatic VLDL overproduction $(12,13)$ and postprandial lipemia (11), we compared postprandial FGF21 responses in two subgroups with normal $(\leq 5 \%)$ and high $(>5 \%)$ liver fat determined by magnetic resonance (MR) spectroscopy (Table 2). This cut point is based on a population study (14).

\section{Determination of liver fat content and abdominal fat volumes}

All subjects underwent an oral fat load test and measurements of subcutaneous and visceral fat depots were measured by MR imaging and liver fat content was measured by MR spectroscopy as described (13). The calculated sum of visceral and subcutaneous fat was expressed as total abdominal fat.

\section{Oral fat load}

After a 12-h fast, the subjects received a mixed meal consisting of bread, butter, cheese, skimmed milk, and tea or coffee ( $50 \mathrm{~g}$ carbohydrates, $72 \mathrm{~g}$ fat (P/S ratio 0.08$)$, and $35 \mathrm{~g}$ protein) in the morning. Blood samples were drawn before and 3, 4, 6, 8, and $10 \mathrm{~h}$ after the meal. During this time, only water was served ad libitum. The subjects remained physically inactive during the whole study.

\section{Separation of lipoproteins and biochemical methods}

Blood samples obtained during the oral fat load study were separated into lipoprotein fractions with gradient

Table 2 Metabolic characteristics and body composition of the study subjects according to liver fat $\leq 5 \%$ or $>5 \%$. Data are mean \pm S.D. or interquartile range.

\begin{tabular}{lll}
\hline & \multicolumn{2}{c}{ Mean \pm s.D. } \\
\cline { 2 - 3 } & LF $\leq 5 \%$ & LF $>5 \%$ \\
\hline Age (years) & $43.3 \pm 12.8$ & $41.0 \pm 8.3$ \\
Gender (males/females) & $15 / 20$ & $7 / 5^{\star}$ \\
BMI (kg/m $\left.{ }^{2}\right)$ & $25.0 \pm 3.9$ & $28.6 \pm 3.6^{\dagger}$ \\
Liver fat (\%) & $1.5(1.98)$ & $12.0(8.9)^{\dagger}$ \\
Subcutaneous fat $\left(\mathrm{cm}^{2}\right)$ & $3429(1893)$ & $5425(3342)^{\dagger}$ \\
Visceral fat $\left(\mathrm{cm}^{2}\right)$ & $1215(1153)$ & $2310(658)^{\dagger}$ \\
Glucose $(\mathrm{mmol} / \mathrm{l})$ & $5.1 \pm 0.6$ & $5.6 \pm 0.5^{*}$ \\
Glycohemoglobin A1C (\%) & $5.5 \pm 0.36$ & $5.6 \pm 0.35$ \\
LDL cholesterol (mmol/I) & $3.45(0.61)$ & $3.28(1.57)$ \\
HDL cholesterol (mmol/l) & $1.48 \pm 0.35$ & $1.23 \pm 0.44$ \\
Triglycerides (mmol/l) & $1.17(0.83)$ & $2.42(2.39)^{\dagger}$ \\
ALT (U/l) & $28(16)$ & $38(32)^{\star}$ \\
\hline
\end{tabular}

${ }^{\star} P<0.05 ;{ }^{\dagger} P<0.01$. 
ultracentrifugation. Concentrations of TG, apoB- 48 and apoB-100, and cholesterol were measured from whole plasma and/or chylomicron (Sf >400), VLDL1 (Sf 60-400), VLDL2 (Sf 20-60), and intermediate density lipoprotein (IDL) (Sf 12-20) fractions at each time point as described (13). FGF21 concentrations were analyzed from serum samples at 0,4 , and $8 \mathrm{~h}$. The serum samples for FGF21 analysis were prepared by centrifugation after blood collection and stored at $-80{ }^{\circ} \mathrm{C}$ until analyzed. FGF2 1 concentrations were measured by ELISA kit (BioVendor GmbH, Heidelberg, Germany) according to the manufacturer's instructions. In statistical analyses, the values below the sensitivity of the assay were assigned a value $0.1 \mathrm{pg} / \mathrm{ml}$. Intra-assay coefficient of variation (CV) was $3 \%$ and inter-assay CV was $12.6 \%$. Plasma FFA and apoA1 concentrations were measured as described previously (13) and $\beta$-hydroxybutyrate was measured by an enzymatic method (DiaSys Diagnostic Systems $\mathrm{GmbH}$, Holzheim, Germany).

\section{Statistical analysis}

Statistical comparisons of data were performed by SPSS Statistical Package (version 19.0, SPSS, Inc., Armonk, NY, USA). Data are presented as mean \pm s.D. or S.E.M. and as medians and ranges, when appropriate. The area under curve (AUC) for postprandial variables was calculated according to the trapezoid rule. The withinsubject change from baseline during postprandial period was analyzed with Greenhouse-Geisser corrected repeated measures ANOVA. We used Shapiro-Wilk's test to explore the normality of variable distribution. Variables were compared between subjects with low liver fat $(\leq 5 \%)$ content and subjects with high liver fat $(>5 \%)$ content using Student's $t$-test or Mann-Whitney $U$ test when assumptions of normal distribution were not met. Correlations were calculated with Spearman's rank correlations method. If significant change was detected, Bonferroni correction was applied in the analysis. A multivariable regression analysis was performed by a stepwise method. We considered a two-tailed $P$ value $<0.05$ as statistically significant.

\section{Results}

\section{Fasting correlations between FGF21 and metabolic parameters}

Fasting FGF21 concentrations demonstrated a large range from undetectable concentrations to $832.7 \mathrm{pg} / \mathrm{ml}$. Fasting FGF21 displayed only a borderline correlation with fasting plasma TG $(r=0.308$, $P=0.06)$, but a significant correlation existed with TG in chylomicron and VLDL1 fractions $(r=0.424$, $P=0.008$ and $r=0.382, P=0.018$ respectively). No significant relationship existed between fasting
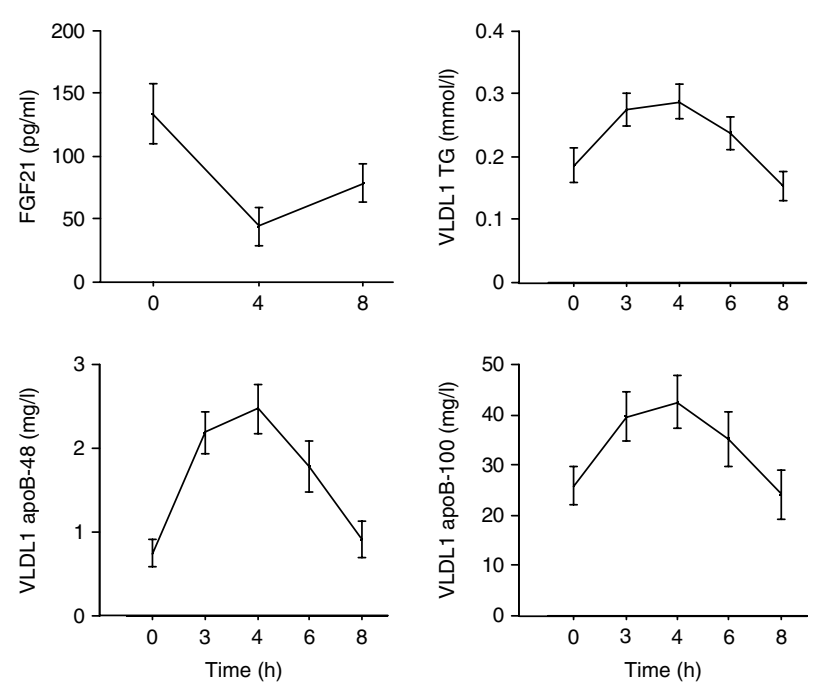

Figure 1 Postprandial changes in concentrations of plasma FGF21 and TG, apoB-48, and apoB-100 in VLDL1 fraction during oral fat load in all study subjects $(n=47)$. Data are mean \pm s.E.M. $P<0.001$ for change from baseline for each parameter by repeated measures ANOVA (Greenhouse-Geisser).

FGF21 and baseline FFA, $\beta$-hydroxybutyrate, apoA1, or apoB concentrations. Fasting FGF21 concentration was strongly related to liver $(r=0.395, P<0.01)$ and visceral $(r=0.342, P<0.05)$ fat, but not to subcutaneous fat.

\section{Postprandial FGF21 and TRL responses}

The FGF21 response to the oral fat load is shown in Fig. 1. Postprandial FGF21 concentration decreased in 46 of the 47 subjects at $4 \mathrm{~h}(P=0.045)$. The concentration returned toward the fasting levels by the end of the study at $8 \mathrm{~h}$. As shown in Fig. 1, FGF21 and TRLs in VLDL1 demonstrated reciprocal patterns after the oral fat load. Besides elevation of TG in plasma and lipoprotein fractions (chylomicron, VLDL1, and VLDL2), significant increases occurred in concentrations of apoB-48 and apoB-100 in each of these TRL fractions $(P<0.01$ by repeated measures ANOVA), indicating that both intestinal and hepatic TRL particles contributed to postprandial lipemia (Fig. 1).

Postprandial TG, apoB-48, and apoB-100 measurements at 3, 4, 6, and $8 \mathrm{~h}$ correlated with FGF21 concentrations (Table 3 ) during the postprandial period (data not shown). To avoid multiple testing, we calculated the correlations between postprandial apoB-48 and apoB-100 AUC values in VLDL1 and VLDL2 fractions and fasting FGF21 and the change of FGF21 from 0 to $4 \mathrm{~h}(\Delta \mathrm{FGF} 21,0-4 \mathrm{~h})$. Although FGF21 levels decreased and TRL levels increased after the oral fat load, the FGF21 was used and therefore the correlations presented in Table 3 are positive. Table 4 reports the multivariable analysis. 
Table 3 Spearman's correlation coefficients for postprandial apolipoprotein B-48 (apoB-48) and apoB-100 responses (area under curve (AUC)) in very low-density lipoprotein 1 (VLDL1) and VLDL2 fractions and for fasting fibroblast growth factor 21 (FGF21; $0 \mathrm{~h})$ and for the change of FGF21 from 0 to $4 \mathrm{~h}(\Delta 0-4 \mathrm{~h})$.

\begin{tabular}{llc}
\hline & \multicolumn{2}{c}{ Spearman's $\rho$ for AUC } \\
\cline { 2 - 3 } & FGF21 $(0 \mathrm{~h})$ & $\Delta$ FGF21 $(0-4 \mathrm{~h})$ \\
\hline B-48 & & \\
VLDL1 & $0.374^{\mathrm{a}}$ & $0.398^{\mathrm{b}}$ \\
VLDL2 & $0.497^{\mathrm{b}}$ & $0.554^{\mathrm{b}}$ \\
B-100 & & \\
VLDL1 & $0.389^{\mathrm{b}}$ & $0.407^{\mathrm{b}}$ \\
VLDL2 & $0.431^{\mathrm{b}}$ & $0.457^{\mathrm{b}}$ \\
\hline
\end{tabular}

${ }^{\mathrm{a} C}$ Correlation is significant at the 0.05 level (two-tailed).

${ }^{\mathrm{b}}$ Correlation is significant at the 0.01 level (two-tailed).

\section{Postprandial FGF21 and TRL responses between subjects with high vs low liver fat}

We observed increased FGF21 levels in subjects with high liver fat compared with those with low liver fat (Table 2 and Fig. 2). These subjects with high liver fat also exhibited significantly higher postprandial responses of apoB-48 and apoB-100 in VLDL1 fraction. Besides, the AUC values for plasma TG $(P=0.008)$, chylomicron TG $(P=0.032)$, VLDL2 TG $(P=0.016)$, and chylomicron apoB-100 $(P=0.007)$ were increased in high liver fat subjects.

\section{Postprandial FGF21 responses and measures of liver, visceral, and subcutaneous fat}

Postprandial FGF21 levels and $\Delta$-change of FGF from 0 to $4 \mathrm{~h}$ correlated positively with liver and visceral fat, but not with subcutaneous fat in the whole study population (data not shown).

\section{Postprandial FGF21 and other metabolic parameters}

Postprandial plasma FFA concentrations demonstrated only insignificant changes, which were opposite to those of TG with slight decrease at $4 \mathrm{~h}$ and increase at $8 \mathrm{~h}$. FGF21 concentrations followed the pattern of FFA concentrations but were not related to FFA at $4 \mathrm{~h}$ or to the $\Delta$-changes of FFA from 0 to $4 \mathrm{~h}$. The 8 -h FFA concentration correlated with the FGF 21 nadir at $4 \mathrm{~h}$ $(r=-0.346, P=0.019)$. We observed no correlations between FGF2 1 and $\beta$-hydroxybutyrate levels at 4 or $8 \mathrm{~h}$ (data not shown).

\section{Discussion}

Our study is the first to report a decrease in postprandial FGF21 concentration in response to an oral fat load in humans. Although FFAs are known regulators of fasting FGF21 expression (4), we did not detect this association during the postprandial period. Our findings indicate that both fasting and postprandial chylomicron and VLDL particles are better predictors of FGF21 concentrations than fasting or postprandial plasma FFAs.

Specifically, the concentrations of FGF21 both in fasting and after oral fat load were positively correlated with postprandial TRL areas in chylomicron, VLDL1, and VLDL2 fractions, i.e. with TRLs and their remnants. The association applied to both apoB-48 and apoB-100 AUC values, which implies that both intestinal and hepatic-derived particles may reflect FGF21 secretion. In multivariate analysis, TG and apoB-48 AUC in VLDL2 fraction, i.e. small TRL remnants, were the best predictors of fasting FGF21 levels and postprandial FGF 21 change from fasting to $4 \mathrm{~h}$. It is possible that the suppression of FGF21 regulates postprandial lipid metabolism and permits a better clearance of TRLs. This obviously remains speculative due to our observational study design and due to antecedent lack of data as to whether FGF21 regulates lipoprotein lipase activity, remnant removal through hepatic receptors, or fatty acid uptake by hepatocytes in humans. Irrespective of the specific mechanism, the decrease in FGF21 following oral fat load was closely related to an increase in postprandial intestinal and hepatic TRLs and their remnants, but to find whether a feedback regulation exists between TRLs and postprandial FGF21, further research is warranted.

The postprandial decrease in FGF21 was a nearly uniform phenomenon among the 47 study participants. Previously, lipid-heparin infusion to healthy men induced a threefold rise in FFA and eightfold rise in TG concentrations, but the FGF21 levels remained unchanged (15). The differences between these findings and ours include the route of fat administration (i.v. vs oral) and the use of heparin, a potent inductor of lipolysis, resulting in considerable rise in plasma FFA. Contrary to the findings of Mai et al. (15), we observed a significant physiological rise in TRLs but only a minor

Table 4 Best predictors of fasting fibroblast growth factor 21 (FGF21) and the change of FGF21 from 0 to $4 \mathrm{~h}(\Delta \mathrm{FGF} 21,0-4 \mathrm{~h})$ using postprandial triglyceride (TG), apolipoprotein B-48 (apoB-48), and apoB-100 area under curve (AUC) values, liver fat, and abdominal fat volumes as independent variables in a stepwise analysis. Adjusted $r^{2}$ represents the combined proportion of variation for FGF21 and $\Delta$ FGF21 $(0-4 \mathrm{~h})$, explained by the predictors remaining in the model. Parameters included in the analysis were: liver fat; subcutaneous fat; intra-abdominal fat; chylomicron AUC for TG, apoB-48, and apoB-100; very low-density lipoprotein 1 (VLDL1) AUC for TG, apoB-48, and apoB-100; and VLDL2 AUC for TG, apoB-48, and apoB-100.

\begin{tabular}{|c|c|c|}
\hline & FGF21 (0 h) & $\Delta$ FGF21 (0-4 h) \\
\hline $\begin{array}{l}\text { Best } \\
\text { predictors }\end{array}$ & $\begin{array}{l}\text { VLDL2 apoB-48 AUC, } \\
\text { VLDL2 TG AUC, } \\
\text { liver fat }\end{array}$ & $\begin{array}{l}\text { VLDL2 apoB-48 AUC, } \\
\text { VLDL2 TG AUC, } \\
\text { liver fat }\end{array}$ \\
\hline Adj. $r^{2}$ & $0.354 ; P<0.001$ & $0.339 ; P<0.001$ \\
\hline
\end{tabular}




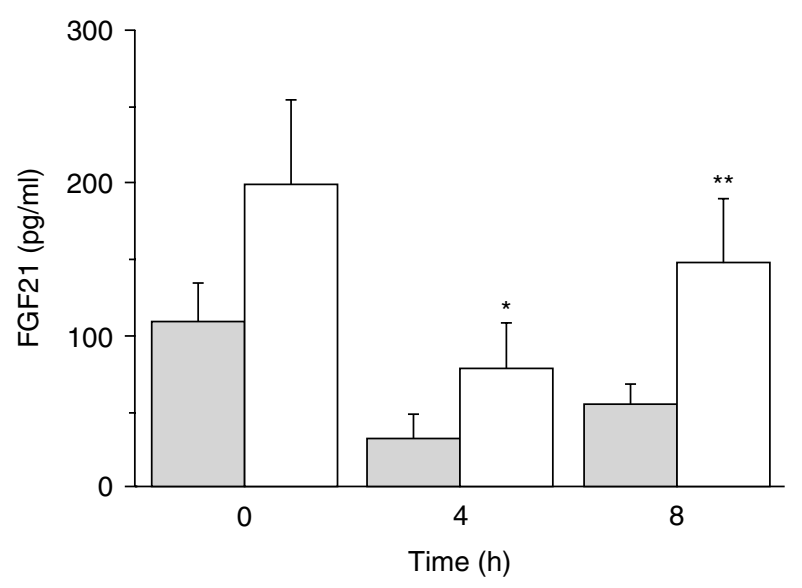

Figure 2 The concentrations of FGF21 at baseline and 4 and $8 \mathrm{~h}$ after the oral fat load in subjects with normal ( $\leq 5 \%$, gray bars) and increased ( $>5 \%$, white bars) liver fat. Data are mean \pm s.E.M. The stars indicate the difference between the groups by MannWhitney $U$ test.

decrease in plasma FFA following an oral fat load, which may explain differences in outcomes. Animal data on metabolic regulation of FG21 are conflicting and not directly applicable to humans. In rats, however, hepatic Ppara and Fgf21 expression and also FGF21 serum levels increased significantly in response to carbohydrate and fat feeding (16) and Fgf21 gene expression was inversely related to hepatic TG concentration (17). Our results may thus imply that FGF21 levels may not follow FFA changes, but reflect the rise in TRL concentrations in the postprandial period and liver fat content, since these two are closely related (13).

Subjects with low levels of postprandial TRL remnants and low liver fat demonstrated low FGF21 levels 4 and $8 \mathrm{~h}$ after an oral fat load. Instead, in apparently healthy subjects with slightly increased liver fat $(>5 \%)$ and more enhanced postprandial lipemia, FGF21 also decreased, but remained significantly higher at postprandial time-points suggesting impaired suppression of postprandial FGF21. Furthermore, multivariate analysis of the whole study population indicated that liver fat content predicted fasting FGF21 levels and postprandial $\Delta \mathrm{FGF} 21$ change from fasting to $4 \mathrm{~h}$. This finding is in line with a recent finding that fasting FGF21 rises progressively with increasing liver fat content (18). Previous studies demonstrated elevated fasting FGF21 levels in metabolic conditions such as obesity, dyslipidemia, and type 2 diabetes mellitus, which are all associated with increased liver fat and NAFLD $(6,10,19)$. Importantly, these conditions also share excessive postprandial lipemia as a common feature and a risk for cardiovascular disease. Especially, high levels and prolonged residence time of small TRL remnants provide a link to atherosclerosis, allowing vascular endothelium to load with excess cholesterol and promoting low-grade inflammation and thrombosis (20). To determine whether impaired postprandial suppression of FGF21 clusters occurs with other markers of atherosclerosis warrants further studies.

The relevance of FGF21 in human metabolism is currently under extensive research activity, and lately FGF21 has been implicated in various clinical conditions including insulin resistance $(8,9)$, NAFLD $(8,9,21)$, mitochondrial diseases (22), anorexia nervosa (23), and as a biomarker predicting type 2 diabetes (24). These apparently heterogenic clinical conditions reflect the fact that the role of FGF21 is poorly characterized in human physiology. Therefore, our results are not readily applicable to the clinic, but expand the significance of FGF21 to postprandial lipid metabolism possibly modified by liver fat content.

Our study population was rather small and it did not receive a weight-based amount of oral fat. Furthermore, correlation analyses do not reflect any cause-and-effect relationship. As we did not measure insulin sensitivity in our subjects, we are unable to estimate whether variation in postprandial FGF21 is accounted for by different degrees of insulin sensitivity, which is the case in the fasting state (9). There is a lack of a fasting control group, but insignificant daytime variations of FGF21 levels have been demonstrated previously (5).

Taken together, the results from this pilot study imply that postprandial TRLs and their remnants are related to FGF21 levels after a fat load. Subjects with increased liver fat exhibit diminished suppression of postprandial FGF21. Our findings suggest that the postprandial accumulation of TRL remnants and liver fat modulate postprandial FGF21 levels.

\section{Declaration of interest}

The authors declare that there is no conflict of interest that could be perceived as prejudicing the impartiality of the research reported.

\section{Funding}

This study was supported by a grant from Helsinki University Central Hospital Research Foundation.

\section{References}

1 Kralisch S \& Fasshauer M. Fibroblast growth factor 21: effects on carbohydrate and lipid metabolism in health and disease. Current Opinion in Clinical Nutrition and Metabolic Care 201114 354-359. (doi:10.1097/MCO.0b013e328346a326)

2 Badman MK, Pissios P, Kennedy AR, Koukos G, Flier JS \& MaratosFlier E. Hepatic fibroblast growth factor 21 is regulated by PPARalpha and is a key mediator of hepatic lipid metabolism in ketotic states. Cell Metabolism 20075 426-437. (doi:10.1016/j. cmet.2007.05.002)

3 Long YC \& Kharitonenkov A. Hormone-like fibroblast growth factors and metabolic regulation. Biochimica et Biophysica Acta 20111812 791-795. (doi:10.1016/j.bbadis.2011.04.002) 
4 Domouzoglou EM \& Maratos-Flier E. Fibroblast growth factor 21 is a metabolic regulator that plays a role in the adaptation to ketosis. American Journal of Clinical Nutrition 201193 901S-905S. (doi:10.3945/ajen.110.001941)

5 Galman C, Lundasen T, Kharitonenkov A, Bina HA, Eriksson M, Hafstrom I, Dahlin M, Amark P, Angelin B \& Rudling M. The circulating metabolic regulator FGF21 is induced by prolonged fasting and PPARalpha activation in man. Cell Metabolism $2008 \mathbf{8}$ 169-174. (doi:10.1016/j.cmet.2008.06.014)

6 Dushay J, Chui PC, Gopalakrishnan GS, Varela-Rey M, Crawley M, Fisher FM, Badman MK, Martinez-Chantar ML \& Maratos-Flier E. Increased fibroblast growth factor 21 in obesity and nonalcoholic fatty liver disease. Gastroenterology 2010139 456-463. (doi:10. 1053/j.gastro.2010.04.054)

7 Yu H, Xia F, Lam KSL, Wang Y, Bao Y, Zhang J, Gu Y, Zhou P, Lu J, Jia W \& Xu A. Circadian rhythm of circulating fibroblast growth factor 21 is related to diurnal changes in fatty acids in humans. Clinical Chemistry 201157 691-700. (doi:10.1373/clinchem. 2010.155184)

8 Zhang X, Yeung DC, Karpisek M, Stejskal D, Zhou ZG, Liu F, Wong RL, Chow WS, Tso AW, Lam KS \& Xu A. Serum FGF2 1 levels are increased in obesity and are independently associated with the metabolic syndrome in humans. Diabetes 200857 1246-1253. (doi:10.2337/db07-1476)

9 Chavez AO, Molina-Carrion M, Abdul-Ghani MA, Folli F, Defronzo RA \& Tripathy D. Circulating fibroblast growth factor-21 is elevated in impaired glucose tolerance and type 2 diabetes and correlates with muscle and hepatic insulin resistance. Diabetes Care 200932 1542-1546. (doi:10.2337/dc09-0684)

10 Yilmaz Y, Eren F, Yonal O, Kurt R, Aktas B, Celikel CA, Ozdogan O, Imeryuz N, Kalayci C \& Avsar E. Increased serum FGF21 levels in patients with nonalcoholic fatty liver disease. European Journal of Clinical Investigation 201040 887-892. (doi:10.1111/j.13652362.2010.02338.x)

11 Matikainen N \& Taskinen M. Postprandial triglyceride-rich lipoproteins in insulin resistance and type 2 diabetes. Future Lipidology 20083 531-543. (doi:10.2217/17460875.3.5.531)

12 Adiels M, Taskinen MR, Packard C, Caslake MJ, Soro-Paavonen A, Westerbacka J, Vehkavaara S, Hakkinen A, Olofsson SO, YkiJarvinen H \& Boren J. Overproduction of large VLDL particles is driven by increased liver fat content in man. Diabetologia 200649 755-765. (doi:10.1007/s00125-005-0125-z)

13 Matikainen N, Manttari S, Westerbacka J, Vehkavaara S, Lundbom N, Yki-Jarvinen H \& Taskinen MR. Postprandial lipemia associates with liver fat content. Journal of Clinical Endocrinology and Metabolism 200792 3052-3059. (doi:10.1210/jc.20070187)

14 Szczepaniak LS, Nurenberg P, Leonard D, Browning JD, Reingold JS, Grundy S, Hobbs HH \& Dobbins RL. Magnetic resonance spectroscopy to measure hepatic triglyceride content: prevalence of hepatic steatosis in the general population. American Journal of Physiology. Endocrinology and Metabolism $2005 \mathbf{2 8 8}$ E462-E468. (doi:10.1152/ajpendo.00064.2004)

15 Mai K, Schwarz F, Bobbert T, Andres J, Assmann A, Pfeiffer AFH \& Spranger J. Relation between fibroblast growth factor-21, adiposity, metabolism, and weight reduction. Metabolism 2011 60 306-311. (doi:10.1016/j.metabol.2010.02.016)

16 Sanchez J, Palou A \& Pico C. Response to carbohydrate and fat refeeding in the expression of genes involved in nutrient partitioning and metabolism: striking effects on fibroblast growth factor-21 induction. Endocrinology $2009 \quad 150 \quad 5341-5350$. (doi:10.1210/en.2009-0466)

17 Uebanso T, Taketani Y, Fukaya M, Sato K, Takei Y, Sato T, Sawada N, Amo K, Harada N, Arai H, Yamamoto H \& Takeda E. Hypocaloric high-protein diet improves fatty liver and hypertriglyceridemia in sucrose-fed obese rats via two pathways. American Journal of Physiology. Endocrinology and Metabolism 2009297 E76-E84. (doi:10.1152/ajpendo.00014.2009)

18 Yan H, Xia M, Chang X, Xu Q, Bian H, Zeng M, Rao S, Yao X, Tu Y, Jia W \& Gao X. Circulating fibroblast growth factor 21 levels are closely associated with hepatic fat content: a cross-sectional study. PLOS ONE 20116 e24895. (doi:10.1371/journal.pone. 0024895)

19 Li H, Fang Q, Gao F, Fan J, Zhou J, Wang X, Zhang H, Pan X, Bao Y, Xiang K, Xu A \& Jia W. Fibroblast growth factor 21 levels are increased in nonalcoholic fatty liver disease patients and are correlated with hepatic triglyceride. Journal of Hepatology 201053 934-940. (doi:10.1016/j.jhep.2010.05.018)

20 Catapano AL, Reiner Z, De Backer G, Graham I, Taskinen MR, Wiklund O, Agewall S, Alegria E, Chapman MJ, Durrington P, Erdine S, Halcox J, Hobbs R, Kjekshus J, Filardi PP, Riccardi G, Storey RF \& Wood D. European Society of Cardiology (ESC). European Atherosclerosis Society (EAS). ESC/EAS Guidelines for the management of dyslipidaemias The Task Force for the management of dyslipidaemias of the European Society of Cardiology (ESC) and the European Atherosclerosis Society (EAS). Atherosclerosis 2011217 3-46. (doi:10.1016/j.atherosclerosis.2011.06.028)

21 Li X, Fan X, Ren F, Zhang Y, Shen C, Ren G, Sun J, Zhang N, Wang W, Ning G \& Yang J. Serum FGF21 levels are increased in newly diagnosed type 2 diabetes with nonalcoholic fatty liver disease and associated with hsCRP levels independently. Diabetes Research and Clinical Practice 201193 10-16. (doi:10.1016/j. diabres.2011.02.034)

22 Suomalainen A, Elo JM, Pietilainen KH, Hakonen AH, Sevastianova K, Korpela M, Isohanni P, Marjavaara SK, Tyni T, Kiuru-Enari S, Pihko H, Darin N, Ounap K, Kluijtmans LA, Paetau A, Buzkova J, Bindoff LA, Annunen-Rasila J, Uusimaa J, Rissanen A, Yki-Jarvinen H, Hirano M, Tulinius M, Smeitink J \& Tyynismaa H. FGF-21 as a biomarker for muscle-manifesting mitochondrial respiratory chain deficiencies: a diagnostic study. Lancet Neurology 201110 806-818. (doi:10.1016/S1474-4422 (11)70155-7)

23 Dostalova I, Kavalkova P, Haluzikova D, Lacinova Z, Mraz M, Papezova H \& Haluzik M. Plasma concentrations of fibroblast growth factors 19 and 21 in patients with anorexia nervosa. Journal of Clinical Endocrinology and Metabolism 200893 3627-3632. (doi:10. 1210/jc.2008-0746)

24 Chen C, Cheung BM, Tso AW, Wang Y, Law LS, Ong KL, Wat NM, $\mathrm{Xu} A$ \& Lam KS. High plasma level of fibroblast growth factor 21 is an Independent predictor of type 2 diabetes: a 5.4-year population-based prospective study in Chinese subjects. Diabetes Care 201134 2113-2115. (doi:10.2337/dc11-0294)

Received 6 September 2011

Revised version received 19 November 2011

Accepted 21 December 2011 